\title{
Towards ps-LIBS tritium measurements in W/Al materials
}

\author{
Arnaud Bultel $^{\mathrm{a}}$, Vincent Morel ${ }^{\mathrm{a}}$, Aurélien Favre ${ }^{\mathrm{a}}$, Gilles Godard ${ }^{\mathrm{a}}$, Abdenacer Benyagoub ${ }^{\mathrm{b}}$, \\ Isabelle Monnet ${ }^{\mathrm{b}}$, Alexandre Sémérok ${ }^{\mathrm{c}}$, Maria Dinescu ${ }^{\mathrm{d}}$, Sabina Markelj ${ }^{\mathrm{e}}$, Philippe Magaud ${ }^{\mathrm{f}}$, \\ Christian Grisolia ${ }^{\mathrm{f}}$
}

\author{
${ }^{a}$ CORIA, UMR CNRS 6614, Normandie University, 76801 Saint-Etienne du Rouvray, France \\ ${ }^{\mathrm{b}}$ CIMAP, UMR 6252, CEA-CNRS-ENSICAEN-UNICAEN, 14070 Caen cedex 5, France \\ ${ }^{\mathrm{c}}$ DEN, CEA Saclay, 91191 Gif-sur-Yvette, France \\ d INFLPR, 77125 Magurele, Romania \\ e Jozef Stefan Institute, 1000 Ljubjana, Slovenia \\ ${ }^{\mathrm{f}}$ IRFM, CEA Cadarache, 13108 Saint-Paul lez Durance, France
}

\section{A R T I C L E I N F O}

\section{Keywords:}

52.38.Mf

52.50.Jm

52.25.Os

\begin{abstract}
A B S T R A C T
This communication deals with experimental results crucial for the sizing of picosecond LIBS measurements dedicated to the determination of tritium density profiles in the plasma facing components. Ablation properties in single pulse on aluminum (considered as a surrogate of beryllium) and tungsten have been obtained for 532 and $1064 \mathrm{~nm}$ picosecond laser pulses. Then, double pulse experiments have been performed to estimate the role played by the second pulse on the spectral radiance observed for lines representative of the ionization degree of the plasma and possible observation of low radiance lines.
\end{abstract}

\section{Introduction}

The in situ determination of the tritium retention in tokamak plasma facing components (PFCs) is crucial in terms of safety due to the activity of this radioisotope $[1,2]$. Besides, the global monitoring of the machine requires this determination to correctly estimate the most appropriate time of aged PFCs decommissioning. The LIBS (Laser-Induced Breakdown Spectroscopy) diagnostic has been proposed to perform such estimates [3]. Under laser irradiation, the PFCs' wall undergoes a solid $\rightarrow$ plasma transition giving rise to the emission of lines whose spectroscopic analysis leads to the multi-elemental composition of the sample provided certain assumptions. Even if most of the LIBS measurements are performed using nanosecond laser pulses with a certain degree of success [3-5], we have chosen to investigate the potentialities of picosecond laser pulses already tested in JET [6]. Previous works have shown that LIBS using ns pulses is not necessarily the most appropriate to the measurement of the concentration of light atoms in heavy matrices. Indeed, the light atoms move fast out of the plasma due to their weaker mass. The number population density of the upper states of the interesting transitions then decreases faster than expected when considering only the expansion and the cooling of the plasma. This behavior leads to an underestimate of their mole fraction in the sample [7]. As a result, the spectroscopic study has to be performed early, when unfortunately the electron density is so high that the Stark broadening is significant and the emission of the radiative recombination continuum prevents a satisfactory observation of the lines. In addition, the overlapping of the hydrogen isotopes lines prevents the precise measurement of their concentration ratio [8]. Moreover, pure thermal effects due to heat transfer within the sample can lead to the modification of the concentration profiles [9]. Using ps pulses leads to lower spectral radiance of the continuum since initial electron density is lower and the spectroscopic analysis can then be performed shortly after the lasermatter interaction with reduced thermal effects. The use of ps laser pulses seems therefore more appropriate to the present case. In addition, it enables the reduction of the ablation depth, but sometimes to the detriment of the signal to noise ratio of the studied lines.

In this paper are summarized the results obtained with the PLEIADES (Plasmas under LasEr IrrADiations and their Experimental Studies) platform available at the CORIA laboratory on samples representative of the materials used as PFCs: tungsten for the divertor part and aluminum considered as a surrogate of beryllium (not used on the platform due to its dust high toxicity) for the remaining part of the inner wall of ITER. Tungsten deposited layers using Plasma Vapor Deposition (WPVD) and ITER-like bulk tungsten (W-ITER) have been studied. First, we report measurements of the ablation rate under different wavelength and focus conditions of the laser pulse. Second, we report the analysis of representative lines of the ionization degree (lines resulting from deexcitation of ions and neutrals on the same wavelength range)

E-mail address: arnaud.bultel@coria.fr (A. Bultel). 
Table 1

Ablation properties of three aluminum and tungsten samples. $a$ is the ablation rate.

\begin{tabular}{llllll}
\hline Sample & $\begin{array}{l}f_{1} \\
(\mathrm{~cm})\end{array}$ & $\begin{array}{l}d_{1} \\
(\mathrm{~cm})\end{array}$ & $\begin{array}{l}E_{1} \\
(\mathrm{~m} J)\end{array}$ & $\begin{array}{l}\lambda_{1} \\
(\mathrm{~nm})\end{array}$ & $\begin{array}{l}a \\
(\mathrm{~nm} / \mathrm{pulse})\end{array}$ \\
\hline $\mathrm{Al}$ & 25 & 1 & 22 & 1064 & 550 \\
$\mathrm{Al}$ & 40 & 1 & 22 & 1064 & 480 \\
$\mathrm{Al}$ & 40 & 1 & 11 & 1064 & 320 \\
W-ITER & 40 & 1 & 11 & 1064 & 80 \\
W-ITER & 40 & 1 & 8 & 1064 & 80 \\
W-ITER & 40 & 1 & 8 & 532 & 250 \\
W-ITER & 40 & 1 & 10 & 532 & 400 \\
W-ITER & 40 & 1 & 16 & 532 & 500 \\
W-PVD & 40 & 1 & 14 & 532 & 100 \\
W-PVD & 40 & 5 & 14 & 532 & 40 \\
W-PVD & 40 & 7 & 14 & 532 & 10
\end{tabular}

when the plasma formed after ablation by the ps laser pulse is reheated by a second ns laser pulse.

\section{The PLEIADES platform}

The PLEIADES platform uses two laser sources available for the experiments in double pulse (DP) configuration. A ps laser source with the wavelength $\lambda_{1}$ is used to ablate the sample placed on a central (x,y,z)-holder after focusing by a devoted lens of $f_{1}$ as focal length. The sample-lens distance is systematically lower than $f_{1}$ to locate the focus point inside the sample and to avoid the breakdown in air. The distance between the sample's surface and the focus point is $d_{1}$ (cf. Table 1). A second laser source is used to produce a ns laser pulse with the wavelength $\lambda_{2}$ delayed by a specific time delay generator. The second pulse is focused in the plasma in recombination using a second lens with $f_{2}$ as focal length. The spectroscopic analysis is performed using an ISOPLANE spectrometer equipped with a $3600 \mathrm{gr} \mathrm{mm}^{-1}$ grating enabling a spectral resolution at least equal to $0.04 \mathrm{~nm}$.

\section{Ablation properties}

The ablation experiments have been performed using ps pulses with different values of energy $\left(E_{1}\right)$ on three types of samples. To test the potential ablation properties of beryllium, aluminum samples have been selected. For tungsten, we have tested bulk ITER-like samples (WITER) and other samples obtained on Cu-Cr-Zr substrates by PVD of a tungsten layer of $\sim 10 \mu \mathrm{m}$ in thickness (W-PVD). The ablation depth has been measured with an optical microscope after a series of 10, 20, 30,40 and 50 pulses along which the ablation regime is linear. The slope $a$ is the ablation rate. Table 1 illustrates the results.

We can see that aluminum is the easiest material to be ablated. WITER is intermediate in terms of ablation rate, followed by W-PVD for which the ablation rate is pretty small. The ablation rate can be significantly reduced by simultaneously decreasing the energy in the pulses and increasing the distance $d_{1}$. In the case of W-PVD, this reduction can reach an order of magnitude. At that time, we do not have a satisfactory explanation of the lower ablation rate observed for W-PVD. Since the spectroscopic analysis requires accumulations of at least 10 pulses to enable a satisfactory signal-to-noise ratio, we can expect a spatial profile resolution belonging to the $[0.1,5] \mu \mathrm{m}$ interval. It is worth to notice that working at $1064 \mathrm{~nm}$ allows a reduction by a factor of 4 of the ablation rate for W-ITER. This wavelength is therefore considered as globally more appropriate to perform LIBS experiments on the concerned materials. In the case of measurements performed on hydrogen isotopes atoms codeposited with Be on W surfaces, this could be coupled with an increase of $d_{1}$ to further reduce the ablation rate.

Due to the reduction of the ablated mass, the typical spectral radiance can be small. Even with an increase of the number of laser pulses accumulations, the signal-to-noise ratio may be weak. In order to keep a

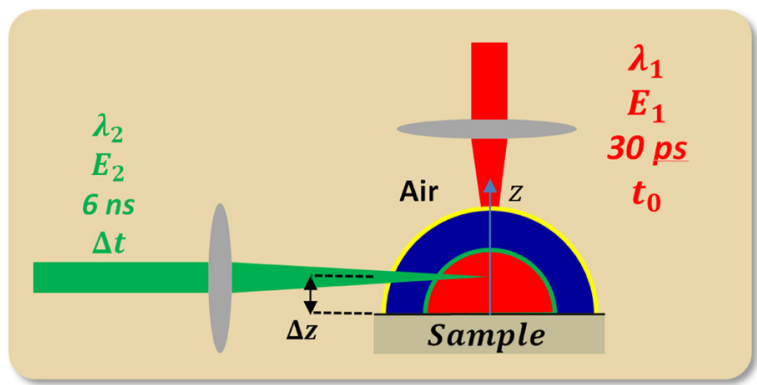

Fig. 1. DP experiments configuration. $d_{1}=1 \mathrm{~mm}$ and $f_{1}=10 \mathrm{~cm}$.

satisfactory spatial resolution, performing measurements under DP regime can be valuable.

\section{DP experiments on aluminum}

The experimental configuration considered in Sections 4 and 5 is given in Fig. 1. The ps pulse (duration $30 \mathrm{ps)} \mathrm{with} E_{1}=20 \mathrm{~mJ}$ and $\lambda_{1}=1064 \mathrm{~nm}$ is focused at time $t_{0}=200 \mathrm{~ns}$ on the sample using a lens of $f_{1}=10 \mathrm{~cm}$ as focal length. The nanosecond pulse (duration $6 \mathrm{~ns}$ ) with $E_{2}=50 \mathrm{~mJ}$ and $\lambda_{2}=532 \mathrm{~nm}$ is tangentially focused (with a lens of $f_{2}=15 \mathrm{~cm}$ as focal length) at a distance $\Delta z$ above the sample's surface in the plasma produced by the first ps pulse. This pulse reaches the sample at time $t_{0}+\Delta t$.

We can easily observe by spectral imagery the temporal evolution of the spectra according to the distance $z$ from the sample's surface. A particularly interesting spectral region corresponds to the resonance doublet lines of $\mathrm{Al}$ around $395 \mathrm{~nm}$. Indeed, a high spectral radiance line of $\mathrm{Al}^{+}$can be observed close to this doublet. Table 2 summarizes the characteristics of the related transitions. Over only a narrow spectral range of $5 \mathrm{~nm}$, the ionization or recombination phase can be directly observed.

Fig. 2 illustrates the spectral imagery obtained for times 50, 200, 250, 300, 350 and $800 \mathrm{~ns}$. The ps laser pulse reaches the aluminum sample at $t_{0}=200 \mathrm{~ns}$. We can easily see the emission of a strong continuum then followed 50 ns later by the emission of the ionic and the neutral lines. In the present case, $\Delta z=2 \mathrm{~mm}$ and $\Delta t=150 \mathrm{~ns}$. Therefore the emission of the continuum is again observed in the aluminum plasma at $t_{0}+\Delta t \simeq 350 \mathrm{~ns}$. The ns laser pulse used for these experiments has a low quality pedestal, which favored the absorption of the laser energy before $350 \mathrm{~ns}$. Afterwards the plasma recombines and only the resonance doublet can still be observed at $800 \mathrm{~ns}$ close to the surface where the plasma is hot and dense enough.

The evolution in time of the net spectral radiance (without continuum) is displayed in Fig. 3 in single pulse and in double pulse regimes for $\Delta z=0$ and $\Delta t=150 \mathrm{~ns}$ or $250 \mathrm{~ns}$. The analysis of these variations has to be performed in the light of the aluminum energy diagram given in Fig. 4. When the second pulse penetrates the plasma, the related photons are absorbed by inverse Bremsstrahlung. Then the electron temperature $T_{e}$ increases, which leads to higher values of the

Table 2

Aluminum and tungsten transitions studied. The energies are given with respect to the neutral ground state.

\begin{tabular}{llllll}
\hline El. & $k \rightarrow i$ & $\begin{array}{l}E_{k} \\
(\mathrm{eV})\end{array}$ & $\begin{array}{l}E_{i} \\
(\mathrm{eV})\end{array}$ & $\begin{array}{l}\lambda_{k i} \\
(\mathrm{~nm})\end{array}$ & $\begin{array}{l}A_{k i} \\
\left(10^{7} \mathrm{~s}^{-1}\right)\end{array}$ \\
\hline $\mathrm{Al}$ & ${ }^{2} S_{1 / 2} \rightarrow{ }^{2} P_{1 / 2}^{0}$ & 3.143 & 0.000 & 394.401 & 4.99 \\
$\mathrm{Al}$ & ${ }^{2} S_{1 / 2} \rightarrow{ }^{2} P_{3 / 2}^{0}$ & 3.143 & 0.014 & 396.152 & 9.85 \\
$\mathrm{Al}^{+}$ & ${ }^{3} F^{0} \rightarrow{ }^{3} D$ & 24.150 & 21.048 & 399.610 & 0.704 \\
$\mathrm{~W}$ & ${ }^{* 0} \rightarrow \rightarrow^{5} D_{4}$ & 5.781 & 0.771 & 247.415 & 11.9 \\
$\mathrm{~W}$ & ${ }_{5}^{* 0} \rightarrow{ }^{3} H_{5}$ & 6.821 & 1.868 & 205.304 & unknown \\
$\mathrm{W}^{+}$ & ${ }^{6} P_{3 / 2}^{0} \rightarrow{ }^{6} S_{5 / 2}$ & 13.764 & 8.784 & 248.877 & 12.6 \\
\hline
\end{tabular}




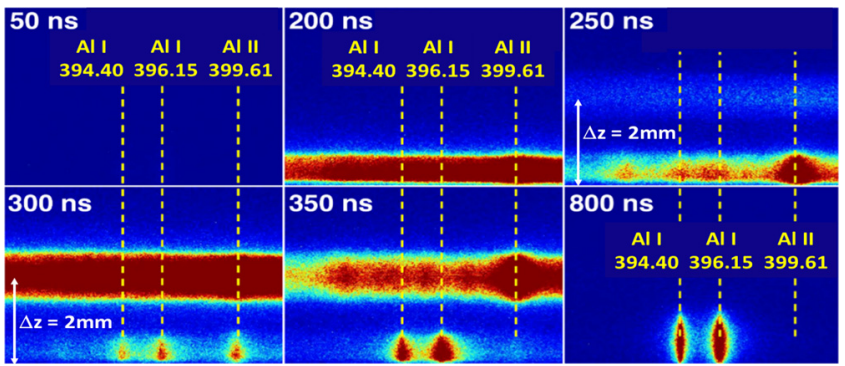

Fig. 2. Spectral imagery obtained on aluminum for $\Delta z=2 \mathrm{~mm}$ and $\Delta t=150$ ns.

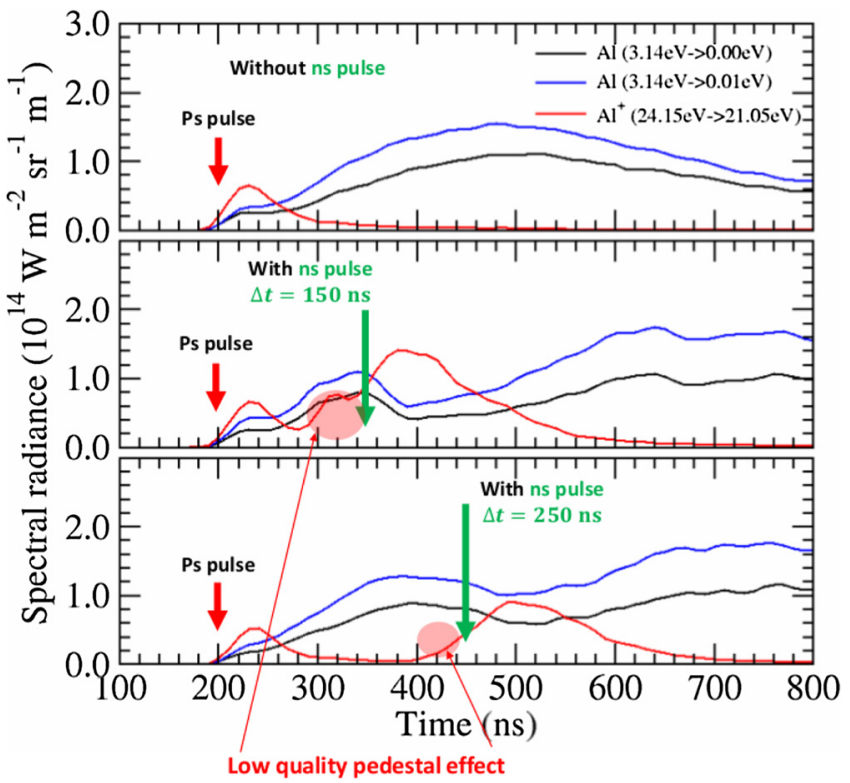

Fig. 3. Net spectral radiance evolutions in single or double pulse configurations observed for the $\mathrm{Al}$ transitions of table 2.

ionization processes rate coefficients. As a result, the electron density $n_{e}$ increases and enhances in turn the absorption by inverse Bremsstrahlung. The concomittant increase in $n_{e}$ and $T_{e}$ induces the decrease in the population density of the neutral excited states, therefore the decrease in the spectral radiance of the related lines. Indeed, since the second laser pulse is tangent to the sample, additional ablation does not take place. This explains the decrease in the spectral radiance of the neutral lines around 360-380 ns for $\Delta t=150$ ns and around 380-480 $\mathrm{ns}$ for $\Delta t=250 \mathrm{~ns}$. The population density of the ionic states increases which leads to an increase in the spectral radiance of the ionic line at the same time. The comparison between $\Delta t=150$ and 250 ns enables the identification of more appropriate conditions for the absorption of the second pulse. The increase in the ionic spectral radiance after the second pulse is higher for $\Delta t=150 \mathrm{~ns}$. Indeed, at this time, $n_{e}$ is higher and the absorption by inverse Bremsstrahlung is more efficient.

\section{DP experiments on tungsten}

Only W-ITER has been studied. The experimental configuration is the same as that of Fig. 1. A time delay of $\Delta t=350 \mathrm{~ns}$ has been chosen. The spectral region $[245,250] \mathrm{nm}$ presents the satisfactory conditions for the direct observation of the influence of the second pulse. Indeed, among numerous lines observable, the two lines (neutral and ionic) reported in Table 2 have a high Einstein coefficient and are easily observed. The spectral imagery displayed in Fig. 5 shows the dynamics induced by the second laser pulse when $\Delta z=2 \mathrm{~mm}$. For aluminum, the absorption takes place on a diameter of $\sim 1 \mathrm{~mm}$. For tungsten, this

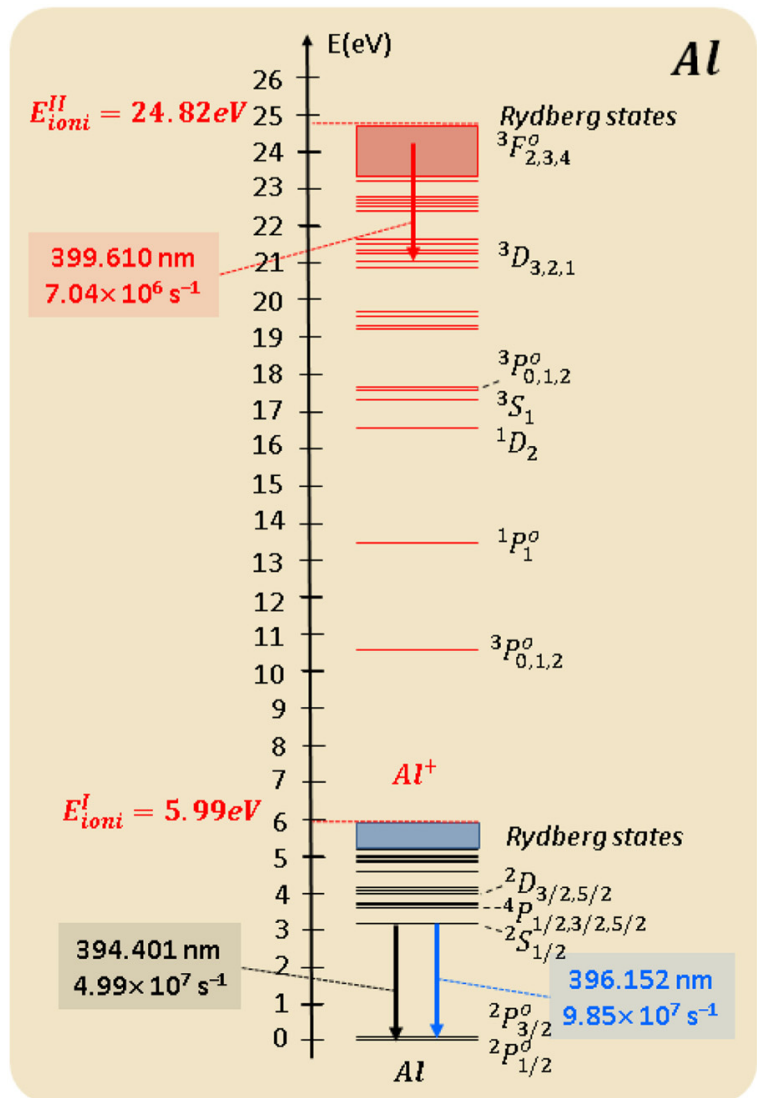

Fig. 4. Energy diagram of aluminum, with the transitions of interest.

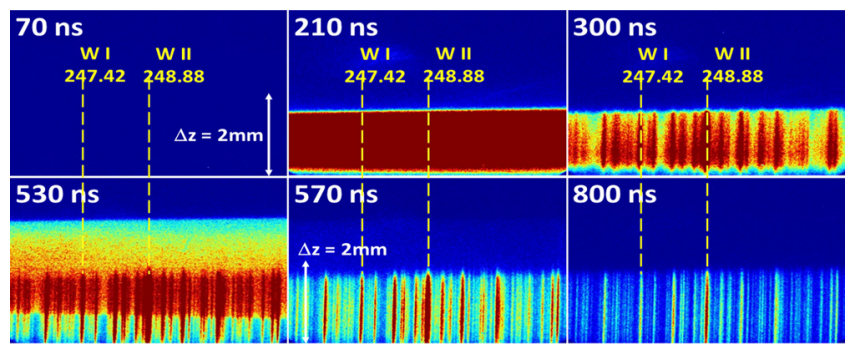

Fig. 5. Same as Fig. 3, but for W-ITER on the $[245,250] \mathrm{nm}$ spectral interval.

diameter is about $2 \mathrm{~mm}$. As a result, one observes the enhanced line broadening induced by the absorption of the ns pulse for $\sim \mathrm{z} \in[1,2]$ $\mathrm{mm}$ at $530 \mathrm{~ns}$. Since the line broadening is only due to the Stark effect resulting from the high value of $n_{e}$, electron density is therefore clearly increased by the ns pulse.

The neutral line at $\lambda=250.304 \mathrm{~nm}$ is weakly perturbed by other lines. Assuming that $T_{e}$ is nearly constant, the Stark broadening depends on $n_{e}$ only. Considering the Full Width at Half Maximum (FWHM) given by $[10]$

$\Delta \lambda_{\mathrm{FWHM}}=2 \omega_{S}\left(\frac{n_{e, m^{-3}}}{10^{22}}\right)$

where $\omega_{S}=6.33 \times 10^{-4} \mathrm{~nm}$ for $T_{e}=10^{4} \mathrm{~K}[11], n_{e}(t)$ has been derived (cf. Fig. 6). When the ns pulse is absorbed by the plasma, the increase in $n_{e}$ is clearly observed. As in the case of aluminum, the low quality of the pedestal leads to the perturbation of $n_{e}$ just before $t_{0}+\Delta t$. Later, around $580 \mathrm{~ns}, n_{e}$ is multiplied by a factor of $\sim 2$. In these conditions, a thorough analysis of the spectral radiance of the lines reported in Table 2 shows that at $t_{0}+600 \mathrm{~ns}$, the spectral radiance of the 247.415 $\mathrm{nm}$ neutral line is multiplied by a factor of 6 whereas the factor is 4 for 


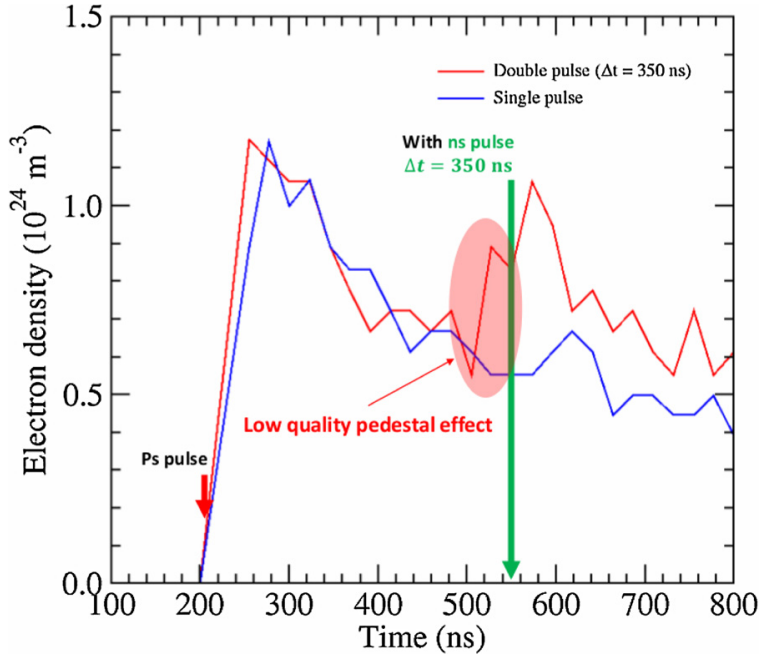

Fig. 6. Evolution of $n_{e}$ derived from the Stark broadening of the tungsten neutral line at $250.304 \mathrm{~nm}$ in single and double pulse.

the $248.877 \mathrm{~nm}$ ionic line. In DP configuration, we can therefore improve the signal-to-noise ratio without additional ablation. This point will be verified in the case the two laser pulses are colinear since this configuration is the only relevant one from the technological point of view in terms of optical access in a tokamak. The way towards spectroscopic observation of lines characterized by very weak radiance is therefore open.

\section{Conclusion}

We have performed preliminary experiments on the PLEIADES platform at the CORIA laboratory leading to the sizing of ps-LIBS on tungsten and aluminum samples. Ablation rates have been measured. Ablation at $1064 \mathrm{~nm}$ is lower than that observed at $532 \mathrm{~nm}$. Using infrared pulses, the depth spatial resolution for the determination of tritium profiles will therefore be at the best of $100 \mathrm{~nm}$, provided the related lines will be separated from others. Double pulse experiments have been performed. In the frame of the configuration used, electron density is increased by a maximum factor of 2 . The spectral radiance of representative lines is increased by a factor of 4 to 6 .

\section{Acknowledgments}

We thank (1) the TRANSAT project funded by the Euratom Research and Training Programme 2014-2018 under grant agreement No 754586, (2) the French Federation for Magnetic Fusion Studies (FRFCM) and the Eurofusion consortium funded by the Euratom Research and Training Programme 2014-2018 and 2019-2020 under grant agreement No 633053 and (3) the French Agence Nationale de la Recherche (ANR), through the programme "Investissement d'Avenir" (ANR-10-LABX-09-01) PICOLIBS project, LabEx EMC ${ }^{3}$.

\section{References}

[1] C. Grisolia, A. Semerok, J.-M. Weulersse, F. LeGuern, S. Fomichev, F. Brygo, P. Fichet, P.-Y. Thro, P. Coad, N. Bekris, M. Stamp, S. Rosanvallon, G. Piazza, In-situ tokamak laser applications for detritiation and co-deposited layers studies, J. Nucl. Mater. 363-365 (2007) 1138-1147, https://doi.org/10.1016/j.jnucmat.2007.01. 169.

[2] J. Roth, E. Tsitrone, T. Loarer, V. Philipps, S. Brezinsek, A. Loarte, G. Counsell, R. Doerner, K. Schmid, O. Ogorodnikova, R. Causey, Tritium inventory in ITER plasma-facing materials and tritium removal procedures, Plasma Phys. Control. Fusion 50 (2008) 103001, https://doi.org/10.1088/0741-3335/50/10/103001.

[3] Z. Hu, N. Gierse, C. Li, P. Liu, D. Zhao, L. Sun, J. Oelmann, D. Nicolai, D. Wu, J. Wu, H. Mao, F. Ding, S. Brezinsek, Y. Liang, H. Ding, G.C.L. Luo, Development of laserbased technology for the routine first wall diagnostic on the tokamak EAST: LIBS and LIAS, Phys. Scr. T170 (2017) 014046, https://doi.org/10.1088/1402-4896/ aa8650.

[4] P. Paris, J. Butikova, M. Laan, M. Aints, A. Hakola, K. Piip, I. Tufail, P. Veis, Detection of deuterium retention by LIBS at different background pressures, Phys. Scr. T170 (2017) 014003, https://doi.org/10.1088/0031-8949/2017/T170/ 014003.

[5] K. Piip, H. van der Meiden, L. Hamarik, J. Karhunen, A. Hakola, M. Laan, P. Paris, M. Aints, J. Likonen, K. Bystrov, J. Kozlova, A. Zaloknik, M. Kelemen, S. Markelj, LIBS detection of erosion/deposition and deuterium retention resulting from exposure to pilot-PSI plasmas, J. Nucl. Mater. 489 (2017) 129-136, https://doi.org/ 10.1016/j.jnucmat.2017.03.044.

[6] A. Semerok, C. Grisolia, LIBS for tokamak plasma facing components characterisation: perspectives on in situ cartography, Nucl. Instr. Meth. Phys. Res. A 720 (2013) 31-35, https://doi.org/10.1016/j.nima.2012.12.042.

[7] L. Mercadier, C. Grisolia, H. Roche, A. Semerok, J. Hermann, C. Pocheau, P.Y. Thro, J.-B. Sirven, J.-M. Weulersse, P. Mauchien, M. Sentis, In situ tritium measurements and control by laser techniques, Fusion Sci. Technol. 60 (2011) 1049-1052, https://doi.org/10.1016/j.jnucmat.2017.03.044.

[8] R. Fantoni, S. Almaviva, L. Caneve, F. Colao, G. Maddaluno, P. Gasior, M. Kubkowska, Hydrogen isotope detection in metal matrix using double-pulse laser-induced breakdown spectroscopy, Spectrochim. Acta Part B 129 (2017) 8-13, https://doi.org/10.1016/j.sab.2016.12.008.

[9] L. Mercadier, A. Semerok, P. Kizub, A. Leontyev, J. Hermann, C. Grisolia, P.Y. Thro, In-depth analysis of ITER-like samples composition using laser-indiced breakdown spectroscopy, J. Nucl. Mater. 414 (2011) 485-491, https://doi.org/10. 1016/j.jnucmat.2011.05.030.

[10] N. Farid, C. Li, H. Wang, H. Ding, Laser-induced breakdown spectroscopic characterization of tungsten plasma using the first, second, and third harmonics of an nd:yag laser, J. Nucl. Mater. 433 (2013) 80-85, https://doi.org/10.1016/j.jnucmat. 2012.09.002.

[11] V. Morel, B. Pérès, A. Bultel, A. Hideur, C. Grisolia, Picosecond libs diagnostics for tokamak in situ plasma facing materials chemical analysis, Phys. Scr. T167 (2016) 014016, https://doi.org/10.1088/0031-8949/T167/1/014016. 